\title{
Rivaroxaban versus Vitamin K Antagonists (Warfarin) based on the triple therapy for left ventricular thrombus after ST-Elevation Myocardial Infarction
}

\section{Zhongfan Zhang \\ Jilin University \\ Qian Zhang \\ Jilin University \\ Ming Qu \\ Jilin University \\ Miao Yu \\ Jilin University \\ Zhenya Jiang \\ Jilin University \\ Delin Li \\ Jilin University}

Daoyuan Si

Jilin University

Wenqi Zhang ( $\nabla$ wenqi@jlu.edu.cn )

Jilin University

\section{Research Article}

Keywords: left ventricular thrombus, Triple therapy, ST-Elevation Myocardial Infarction, Rivaroxaban

Posted Date: June 2nd, 2021

DOI: https://doi.org/10.21203/rs.3.rs-571390/v1

License: (9) This work is licensed under a Creative Commons Attribution 4.0 International License. Read Full License

Version of Record: A version of this preprint was published at Heart and Vessels on August 22nd, 2021.

See the published version at https://doi.org/10.1007/s00380-021-01921-z. 


\section{Abstract}

Background: Left ventricular thrombus(LVT) can lead to serious complications, and mostly formed after ST-Elevation myocardial infarction(STEMI). The Off-label use of new oral anticoagulants(NOACs) in the triple therapy of LVT after STEMI has increased in the past few years. As one of the most widely used NOACs, the data of safety and efficacy of rivaroxaban in LVT after STEMI is limited and warrants continued exploring.

Methods: We conducted a retrospective cohort study involving STEMI patients underwent primary percutaneous coronary intervention (PCI). Among patients who developed LVT after STEMI, we evaluated the efficacy and safety of rivaroxaban plus DAPT therapy associated with thrombus resolution and clinical adverse events, compared with triple therapy with VKA.

Results: In 1,335 patients with STEMI, a total of 77 (5.7\%) developed LVT over the follow up period (median 25.0 months). Of the patients diagnosed with LVT, 31 patients were started on triple therapy with VKA, 33 patients on triple therapy with rivaroxaban. There was a consistent similarity in LVT resolution with rivaroxaban application compared to VKA application during the follow-up period[HR: $1.57(95 \% \mathrm{Cl}$ 0.89-2.77), $p=0.096$; Adjusted HR:1.70(95\% Cl 0.90-3.22), $p=0.104]$. When the analysis focused on LVT resolution at different time points during the follow-up period, triple therapy with rivaroxaban showed quicker resolution than with VKA(6months: $p=0.049 ; 12$ months: $p=0.044 ; 18$ months: $p=0.045)$. Meanwhile, similar risks of ISTH bleeding were recorded in both groups, with no difference between the two groups(Rivaroxaban $6.1 \%$ vs VKA 9.7\%, p=0.444). Fewer net adverse clinical events(NACE) were observed in the rivaroxaban group compared with the VKA group[Rivaroxaban $24.2 \%$ vs VKA $58.1 \%$; HR:0.31(95\% Cl 0.14-0.68), $p=0.003$; Adjusted HR: 0.23(95\%Cl 0.09-0.57), $p=0.001]$.

Conclusions: This observational study suggests triple therapy with rivaroxaban has similar and quicker LVT resolution in patients with LVT after STEMI, compared with triple therapy with VKA, and perhaps was accompanied with a better clinical benefit. Larger sample sizes and randomized controlled trials are needed to confirm this observation.

\section{Introduction}

Left ventricular thrombus(LVT) can lead to serious complications after acute myocardial infarction(AMI), which is associated with a higher incidence of embolism(22.2\%), death(18.9\%), and major adverse cardiovascular events(37.1\%)[1]. The current view is that LVT mostly formed after ST-Elevation myocardial infarction(STEMI). Although the generalization and progress of reperfusion therapy used in the early stages of AMI largely reduce the frequency of LVT, the prevalence of LVT in patients with AMI observed in different centers still remains 4\%-15\%[2-7]. In the current guidelines, the first-line therapy recommends conventional triple anticoagulation [Vitamin K Antagonists(VKA) plus dual antiplatelet therapy (DAPT)] for LVT after STEMI[8-10]. However, the Off-label use of new oral anticoagulants(NOACs) in the triple therapy of LVT after STEMI has increased in the past few years. In 
previous studies on LVT, NOACs were usually considered as a whole and directly compared with VKA, which may be inappropriate due to the different anticoagulant mechanisms and pathways of NOACs. As one of the most widely used NOACs, safety and efficacy data of triple therapy with rivaroxaban in the setting of LVT after STEMI is limited.

The major purposes of this study were to evaluate the efficacy and safety of Triple therapy with rivaroxaban in the treatment of LVT after STEMI utilizing clinical outcomes. Triple therapy with VKA was compared as standard treatment.

\section{Methods}

\section{Study population and design}

In this single-center retrospective study, all patients were identified from STEMI patients underwent primary percutaneous coronary intervention (PCI) in the China-Japan Union Hospital of Jilin University from January 2016 to January 2019(Fig. 1). Triple therapy was defined as dual antiplatelet therapy (DAPT) plus oral anticoagulants. DAPT included low-dose aspirin therapy $(100 \mathrm{mg} / \mathrm{d})$ and a P2Y12 inhibitor(clopidogrel $75 \mathrm{mg} / \mathrm{d}$ or ticagrelor $90 \mathrm{mg}$ bid). Triple therapy was started when LVT was diagnosed by transthoracic echocardiography (TTE), and the use of VKA or rivaroxaban was determined by physicians at that time. The dose of rivaroxaban depended on patient-specific factors (i.e creatinine clearance, bleeding risk, age). According to ACC/AHA guidelines, warfarin dose was titrated to maintain an internationally standardized ratio (INR) goal of 2.0 to 2.5[8]. The exclusion criteria for LVT patients is that 1)rivaroxaban or VKA is currently being used for other diseases; 2) using other antithrombotic therapies in patients with LVT(i.e dabigatran, apixaban); 3) severe bleeding tendency; 4) hemorrhage or stroke occurred during hospitalization prior to baseline TTE; and 5)the quality of TTE was suboptimal, or it was not performed after admission.

\section{Transthoracic echocardiography}

All patients underwent screening echocardiography on admission and at follow-up.LVT observed by TTE was defined as 1) a high-density echo mass in the left ventricular lumen, which is different from that of the myocardium; 2) Obvious contour towards the cavity; 3 ) Could be separated from the endocardial layer; 4) exist throughout the cardiac cycle[3,11]. All TTE images were interpreted by two experienced echocardiographists from the cardiac center. A predesignated third echocardiogram physician was consulted to prevent inconsistencies.

\section{Eendpoints and definitions}

The primary efficacy endpoint was defined as the total resolution of LVT during the follow-up period. The secondary endpoint was a composite of net adverse clinical events(NACE) to assess the net clinical benefit, consisting of all-cause mortality, systemic embolism(SE), rehospitalization for cardiovascular events, bleeding. SE is defined as abrupt vascular insufficiency with clinical or radiographic evidence. The safe endpoint is bleeding. According to the International Society of Thrombosis and Hemostasis (ISTH) 
definition[12,13], all bleeding events are classified as minor, clinically relevant non-major (CRNM), or severe bleeding.

\section{Statistical Analysis}

Continuous variables that are normally distributed were expressed as the mean \pm standard deviation (SD), and the t-test was used for comparison. Continuous variables that are Non-normally distributed were expressed as the median (quaternary series) [M (Q1 Q3)], and the Mann-Whitney U test is used for comparison. Fisher exact test or Chi-square testis used to compare categorical variables.

Time-to-event data are presented as Kaplan-Meier method and assessed by the log-rank test. Baseline variables that were considered clinically relevant or that associated at univariate analysis $(P<0.05)$ with outcome were entered into multivariate Cox proportional-hazards regression models to adjusted models. The included Variables were carefully chosen, given the number of events available, to ensure parsimony of the final models[14]. Data analyses were performed using SPSS version 24.0 (IBM Corp, Armonk, NY, USA) and GraphPad Prism 7.00 software (San Diego, California). All P values had a 2-sided significance level of $5 \%$.

\section{Results}

\section{Patients.}

A total of 77 (5.7\%) patients with STEMI were identified to develop LVT. Not surprisingly, of these patients with LVT after STEMI, the majority (91.0\%) presented with anterior infarcts (71 patients). The value of fibrinogen is higher in LVT patients $(p=0.002)$. In terms of echocardiography, LVT patients were significantly lower left ventricular ejection fraction (LVEF) than those with no LVT $(41.0 \% \mathrm{vs} 60.0 \%$, $\mathrm{p} \varangle 0.001)$ and tended to have larger left ventricular end-diastolic dimension(LVEDD)(56.0mmvs46.0mm, $\mathrm{P} \llbracket 0.001)$ (Table 1). More patients with LVT have left ventricular aneurysm(LV aneurysm) (19.5\%vs6.7\%,p®0.001). And in the whole cohort study, 30 patients with LVT (39.0\%) did not undergo catheter-based revascularization therapy within 12 hours of STEMI onset because of late presentation at the hospital, etc.(p凶0.001). The post-PCI TIMI flow grade of 6 LVT patients(7.8\%) was less than or equal to 2(P = 0.013). Of the patients diagnosed with LVT, 31 patients were started on triple therapy with VKA, among which 7 patients failed to meet the therapeutic target of INR, 33 patients on triple therapy with rivaroxaban, and 13 patients on other therapies due to various reasons. Both triple therapy groups were well matched in terms of baseline characteristics except for Hypertension $(p=0.006)$ and LV aneurysm $(p$ $=0.027)$ (Table 2$)$. 
Table 1

Clinical characteristics in patients with and without left ventricular thrombus

\begin{tabular}{llll} 
Characteristics & No LVT & LVT & p Value \\
\cline { 2 - 4 } & $(n=1258)$ & $(n=77)$ &
\end{tabular}

\section{Baseline characteristics}

\begin{tabular}{llll}
\hline Male,n(\%) & $924(73.4)$ & $59(76.6)$ & 0.540 \\
\hline Age,yrs & $62.0(53.0-69.0)$ & $60.0(53.5-68.5)$ & 0.594 \\
\hline Hypertension,n(\%) & $556(44.2)$ & $26(33.8)$ & 0.073 \\
\hline Diabetes mellitus,n(\%) & $236(18.8)$ & $14(18.2)$ & 0.900 \\
\hline Current smoker,n(\%) & $700(55.6)$ & $41(53.2)$ & 0.681
\end{tabular}

\section{Blood examinations on admission}

WBC $\left(\times 10^{9}\right)$

Platelets $\left(\times 10^{9}\right)$

MPV (fl)

Fibrinogen (mg/dl)

Echocardiography

LVEF,\%

$\operatorname{LVEDD}(\mathrm{mm})$

LV aneurysm,n(\%)

\section{Procedural characteristics}

Symptom to balloon time $\geq 12 \mathrm{~h}$

Multivessel disease, $\mathrm{n}(\%)$

Post-PCI TIMI flow grade $\leq 2, \mathrm{n}(\%)$

WBC:white blood cell count; MPV:mean platelet volume; LVEF:left ventricular ejection fraction; LVEDD:left ventricular end-diastolic dimension; LV aneurysm:left ventricular aneurysm;

TIMl:thrombolysis in myocardial infarction; PCl:percutaneous coronary intervention;

\begin{tabular}{lll}
\hline $10.0(8.0-12.0)$ & $11.0(8.0-13.0)$ & 0.105 \\
\hline $216.0(181.0-256.0)$ & $229.0(164.0-266.0)$ & 0.363 \\
\hline $10.0(9.0-10.0)$ & $10.0(9.0-10.5)$ & 0.232 \\
$3.0(3.0-4.0)$ & $4.0(3.0-5.0)$ & 0.002
\end{tabular}

60.0(50.0-65.0)

$41.0(31.0-49.5)$

$₫ 0.001$

$46.0(42.0-49.0)$

$52.0(48.0-58.0)$

$\nabla 0.001$

84(6.7)

15(19.5)

$\bigotimes 0.001$

291(23.1)

30(39.0)

$\varangle 0.001$

42(3.9)

4(5.2)

0.571

29(2.3)

6(7.8)

0.013 
Table 2

Clinical Characteristics in Patients with LVT who received triple anticoagulation (Rivaroxaban/Warfarin ) Characteristics

\begin{tabular}{ll} 
Warfarin & Rivaroxaban \\
\hline$(n=31)$ & $(n=33)$
\end{tabular}

p Value

\section{Baseline characteristics}

\begin{tabular}{llll} 
Male,n(\%) & $23(74.2)$ & $24(72.7)$ & 0.894 \\
\hline Age,yrs & $61.3 \pm 9.0$ & $60.3 \pm 14.7$ & 0.730 \\
\hline Hypertension,n(\%) & $11(33.5)$ & $23(69.7)$ & 0.006 \\
\hline Diabetes mellitus,n(\%) & $5(16.1)$ & $10(30.3)$ & 0.181 \\
\hline Current smoker,n(\%) & $17(54.8)$ & $11(33.3)$ & 0.083 \\
\hline Blood examinations on admission & & & 0.914 \\
\hline WBC $\left(\times 10^{9}\right)$ & $11.0(8.0-13.0)$ & $11.4(8.4-14.0)$ & 0.405 \\
\hline Platelets $\left(\times 10^{9}\right)$ & $229.0(154.0-304.0)$ & $258.0(193.5-307.5)$ & 0.416 \\
\hline MPV (fl) & $10.0(9.0-11.0)$ & $9.6(9.0-10.7)$ & 0.213
\end{tabular}

\section{Echocardiography}

\begin{tabular}{llll} 
LVEF,\% & $41.4 \pm 10.8$ & $42.9 \pm 13.1$ & 0.614 \\
\hline LVEDD $(\mathrm{mm})$ & $52.5 \pm 6.5$ & $51.1 \pm 7.4$ & 0.437 \\
\hline LV aneurysm,n(\%) & $11(35.5)$ & $4(12.1)$ & 0.027
\end{tabular}

\section{Procedural characteristics}

\begin{tabular}{llll} 
Symptom to balloon time $\geq 12 \mathrm{~h}$ & $12(38.7)$ & $15(45.5)$ & 0.585 \\
\hline Multivessel disease,n(\%) & $3(9.7)$ & $1(3.0)$ & 0.272 \\
\hline Post-PCI TIMI flow grade $\leq 2, \mathrm{n}(\%)$ & $2(6.5)$ & $3(9.1)$ & 0.694
\end{tabular}

WBC:white blood cell count; MPV:mean platelet volume; LVEF:left ventricular ejection fraction; LVEDD:left ventricular end-diastolic dimension; LV aneurysmn:left ventricular aneurysm;

TIMl:thrombolysis in myocardial infarction; PCl:percutaneous coronary intervention;

\section{Clinical results}

26 patients $(78.8 \%)$ in the rivaroxaban group and 23 patients $(74.2 \%)$ in the VKA had resolution of LVT over the follow-up up period(Table 3). There was a consistent similarity in LVT resolution with 
rivaroxaban application compared to VKA application during the follow-up period(Fig. 2; Table 3). When the analysis focused on LVT resolution at different time points during the follow-up period, triple therapy with rivaroxaban showed quicker resolution than with VKA(6months: $p=0.049 ; 12$ months: $p=0.044$; 18months: $p=0.045)$ (Fig. 3).

Table 3

Clinical Outcomes during the follow-up period

\begin{tabular}{|lllll|}
\hline Outcomes & Warfarin & Rivaroxaban & $\begin{array}{c}\text { Adjusted } \\
\text { HR(95\%Cl) }\end{array}$ & Value \\
\hline & $(\mathbf{n = 3 1 )}$ & $\mathbf{( n = 3 3 )}$ & & \\
\hline LVT resolution,n(\%) & $23(74.2)$ & $26(78.8)$ & $1.70(0.90-3.22)$ & 0.104 \\
\hline NACE,n(\%) & $18(58.1)$ & $8(24.2)$ & $0.23(0.09-0.57)$ & 0.001 \\
\hline SE,n(\%) & $4(12.9)$ & $1(3.0)$ & $0.29(0.02-3.13)$ & 0.305 \\
\hline $\begin{array}{l}\text { Rehospitalization for cardiovascular } \\
\text { events,n(\%) }\end{array}$ & $12(38.7)$ & $7(21.2)$ & $0.28(0.09-0.83)$ & 0.022 \\
\hline $\begin{array}{l}\text { All-cause mortality } \\
\text { Bleeding events, } n(\%)\end{array}$ & $4(12.9)$ & $1(3.0)$ & $0.226(0.02-$ & 0.234 \\
\hline $\begin{array}{l}\text { Major bleeding,n(\%) } \\
\text { Minor bleeding,n(\%) }\end{array}$ & $3(9.7)$ & $2(6.1)$ & $0.48(0.73-3.20)$ & 0.444 \\
\hline $\begin{array}{l}\text { LVT:left ventricular thrombus; NACE:net adverse clinical events, consisting of all-cause mortality, SE } \\
\text { rehospitalization for cardiovascular events, bleeding; SE: systemic embolism; }\end{array}$ & - \\
\hline
\end{tabular}

With respect to NACE, rates of NACE were high in LVT patients(45.4\%). NACE rates differed significantly between the two triple therapy groups (VKA $58.1 \%$ vs Rivaroxaban $24.2 \%$ ) over the follow-up period(Fig. 4; Table 3). Triple therapy with VKA had a higher rehospitalization rate due to cardiovascular events $(38.7 \% \mathrm{vs} 21.2 \%, \mathrm{p}=0.022)$. The rates of systemic embolism(SE) were lower in triple therapy with rivaroxaban (3.0\% vs $12.9 \%$ ), but it was not statistically significant(Table 3 ). No systemic embolic events occurred in patients within the time of triple therapy with rivaroxaban, with 2 events occurring in patients on VKA. When not in the triple therapy period, 1 event occurred in the rivaroxaban group and 2 events occurred in the VKA group. Meanwhile, similar risks of ISTH bleeding were recorded in both groups, with no difference between the two groups(Rivaroxaban $6.1 \%$ vs VKA 9.7\%,p $=0.444$ ). These events included gastrointestinal bleeding ( $n=1$, VKA group), and gingival bleeding $(n=4$; VKA group, 2; Rivaroxaban group,2). All bleeding events occurred within the time of triple therapy. No major bleeding events were seen in the rivaroxaban triple treatment group.

\section{Discussion}


The major findings of this retrospective study are: 1) the prevalence of LVT in STEMI patients underwent $\mathrm{PCl}$ at our center was 5.7\%; 2)Low LVEF, large LVEDD, LV aneurysm, high hibrinogen, delayed revascularization, and the poor blood flow after revascularization are relevant factors for LVT; 3)there were similar and quicker resolution of LVT in the triple therapy with rivaroxaban compared to patients treated with VKA; 4)the incidence of bleeding was similar in both triple therapy; 5)triple therapy with rivaroxaban had less net adverse clinical events(NACE) during the follow-up period, compared with VKA group.

Triple therapy with VKA is currently considered first-line therapy for LVT after STEMI[8-10]. But the need for dietary restrictions, frequent monitoring, narrow treatment scope, and multiple drug interactions associated with VKA has contributed to an increase in NOACs application worldwide. In previous studies on LVT[15-18], NOACs were usually considered as a whole and directly compared with VKA, which may be inappropriate due to the different anticoagulant mechanisms and pathways of NOACs. To the best of our knowledge, there are no comparative studies regarding triple therapy with single NOACs versus VKA in patients with LVT following STEMI. As one of the most widely used NOACs, the safety and efficacy data of rivaroxaban for LVT after STEMI are limited and warrant continued investigation. Meanwhile, several landmark trials $[19,20]$ have shown rivaroxaban was non-inferior in efficacy, compared to VKA, in preventing thromboembolism for atrial fibrillation(AF). Rivaroxaban also appears to have a positive effect in the treatment of left atrial appendage thrombosis[21]. The formation of LVT is pathologically similar to the formation of left atrial appendage thrombosis, occurring in a low-flow setting. Given the efficacy of rivaroxaban in the prevention of atrial fibrillation thromboembolism and for treatment of left atrial appendage thrombosis, it is reasonable to extrapolate its efficacy to LVT treatment.

The rate of LVT resolution during treatment is one of the most concerned problems. Previous case studies have demonstrated the efficacy of NOACs in patients with LVT after AMI, and a review of all cases shows the safety of these drugs[22-25]. Based on previous case studies and reviews, Daniel a Jones et al.[17]demonstrated that LVT resolution was quicker in the NOACs group than in the VKA group (1-year $82 \%$ vs $64.4 \%, p=0.0018$ ). However, in their study, all NOACs were analyzed as a whole, which may be inaccurate to determine the effect of various NOACs on LVT after AMI. In the present study, only rivaroxaban was used as the NOACs. When LVT resolution rates were analyzed throughout the follow-up period, triple therapy with rivaroxaban or VKA was found to have similar resolution rates. Given the long follow-up time of this study, similar LVT resolution between the two groups are understandable. The difference became apparent when we analyzed the resolution of the two groups using different time points, whether at 6,12 , or 18 months, rivaroxaban had higher resolution than VKA, which supports the efficacy of rivaroxaban, as compared with warfarin, was favorable. However, compared with other studies[15-17], the resolution rate of LVT in the triple therapy of VKA in our center seems to be low. When focusing on this, it is found that this may be related to the INR compliance rate in the triple therapy. Among the patients receiving the triple therapy of VKA, 7 patients $(22.5 \%)$ failed to meet the therapeutic target of INR, which may affect the resolution rate of LVT. 
The clinical issue associated with LVT is the increased risk of SE, with the prevalence of embolization complications in LVT patients ranging from $10-35 \%[3,26]$. In the present study, the incidence $(11.3 \%)$ on VKA plus DAPT appears consistent with earlier publications. The incidence(3.3\%) on rivaroxaban plus DAPT was lower than previous studies on NOACs[16,17]. The study published by Daniel A Jones et al. [17]showed that in the treatment of LVT, no difference in rates of SE was seen between VKA group and NOACs group in patients with LVT after AMI. However, Austin A. Robinson et al.[16]published an observational study of 514 patients with LVT (all indications) showed the use of NOACs was significantly associated with systemic embolism compared with VKA. There may be several possible reasons for the inconsistent results of these studies:1) The diseases included in the same studies were different, and the treatment after the occurrence of LVT in a certain disease was not analyzed separately. 2) All NOACs were analyzed as a whole, without considering the different mechanisms and pathways of action of NOACs, as well as the differences in efficacy and side effects; 3 ) There were different antithrombotic treatment regimens in these same studies, some were given triple therapy(anticoagulant + dual antiplatelet therapy,some were given double therapy(anticoagulation + antiplatelet therapy), and some were given only anticoagulant therapy, which may lead to an imprecise assessment of the pros and cons of treatment options.Although these studies reflect some of the advantages and disadvantages of NOACs as a whole in the treatment of LVT, more precise studies are worth continuing. Rivaroxaban was the only NOACs that was focused on in this study. Previous studies have demonstrated the superior performance of the triple therapy given rivaroxaban in reducing cardiovascular events in patients with atrial fibrillation and ACS[19,20,27]. In the present study, in patients with LVT after STEMI, the triple therapy given rivaroxaban also had a lower incidence of SE than the triple therapy given VKA $(3.0 \%$ vs $12.9 \%$ ),but it was not statistically significant. This may be related to the relatively small sample size in our study. According to previous studies on rivaroxaban, we tend to think that rivaroxaban has a positive effect on SE in patients with LVT after STEMI, which needs to be confirmed in larger clinical trials.

The main disadvantage of continuous anticoagulation is the increased risk of bleeding. The incidence of adverse outcomes such as death, stroke, and reinfarction was higher in patients with bleeding events[28]. Especially in the patients taking antiplatelet agents plus VKA, anticoagulation is associated with increased risk of bleeding[29]. Several studies[30,31] have reported that triple therapy with VKA has a 3-4 times higher risk of major bleeding than antiplatelet therapy alone or single oral anticoagulant.Andrade et al. [31]conducted a systematic review and meta-analysis of the risk of bleeding in patients receiving triple therapy with VKA after PCI. In their analysis, at 30 days and 6 months the rates of major bleeding with DAPT plus VKA were significantly higher than the DAPT. In the WOEST trial[32], 12.3\% of patients receiving triple therapy with VKA had moderate and severe bleeding events within 1 year, significantly higher than those receiving the dual therapy. However, the results observed in the current study of rivaroxaban are different. In the PIONEER AF-PCI trial with non-valvular AF patients who had undergone $\mathrm{PCI}[20]$, the rate of bleeding on triple therapy with rivaroxaban was significantly lower than triple therapy with VKA( $18.0 \%$ vs. $26.7 \%$ ). In the ATLAS ACS 2-TIMI 51 trial among STEMI patients, triple therapy with rivaroxaban reduced the primary efficacy endpoint of cardiovascular death, myocardial infarction, or stroke, but the rate of fatal hemorrhage was not significantly increased $(0.2 \%$ vs. $0.1 \%, p=0.51)$. In the 
present study, similar bleeding risks were documented between the two groups, and showed no difference. But when focusing on NACE consisting of bleeding, all-cause mortality, SE, rehospitalization for cardiovascular events, we found that NACE were lower in triple therapy with rivaroxaban during the follow-up period, compared with triple therapy with VKA. These results are reassuring, because it indicated that the increase in bleeding seen does not offset the benefit of reduction in vascular events, which suggested that triple therapy with rivaroxaban may have a better net clinical benefit for patients with LVT after STEMI.

\section{Limitations}

Firstly, due to the condition of STEMI patients, the diagnosis of LVT was mainly based on echocardiography in this study, which may have lower detection sensitivity and specificity compared with other imaging methods. Secondly, considering the high risk of triple therapy with VKA in previous studies, physicians at our center were very cautious in adjusting the dose of VKA, which may lead to failure to reach the treatment goal of INR, thus affecting the resolution of LVT. Thirdly, the main limitations of this study are the small sample size and non-randomness, albeit this study including the largest number of patients treated with triple therapy with rivaroxaban for assessing LVT after STEMI so far. Therefore, our results should be considered exploratory rather than definitive.

\section{Conclusion}

This observational study suggests triple therapy with rivaroxaban had similar and quicker LVT resolution in patients with LVT after STEMI, compared with triple therapy with VKA, and perhaps was accompanied with a better clinical benefit. Larger sample sizes and randomized controlled trials are needed to confirm this observation.

\section{Abbreviations}

LVT:left ventricular thrombus; STEMI:ST-Elevation myocardial infarction; PCl: percutaneous coronary intervention; VKA:Vitamin K Antagonists; TTE: transthoracic echocardiography WBC:white blood cell count; MPV:mean platelet volume; LVEF:left ventricular ejection fraction; LVEDD:left ventricular enddiastolic dimension; LV aneurysm:left ventricular aneurysm; TIMI:thrombolysis in myocardial infarction; PCl:percutaneous coronary intervention;

\section{Declarations}

\section{Ethics approval and consent to participate}

This retrospective study was reviewed and approved by the ethical review board of China-Japan Union Hospital of Jilin University. All procedures performed in this study involving human participants were in accordance with the ethical standards of the institution and with the 1964 Helsinki Declaration and its 
later amendments or comparable ethical standards. The written informed consent was obtained from each participant on admission.

\section{Availability of data and materials}

The datasets used and/or analyzed in this study are available from the corresponding author upon reasonable request.

\section{Consent for publication}

None.

\section{Competing interests}

The authors have no conflict of interest to disclose.

\section{Funding}

This work was supported by grants from Science and technology project of Jilin Provincial Department of Education $₫ J J K H 20190062 K J \rrbracket$ and Science and Technology of Jilin Province (20180520054JH and $20200801076 \mathrm{GH}$ )

\section{Author Contributions}

ZZ, DS, and WZ conceived and designed the study. ZZ and QZ collected and analyzed data, performed the literature search. ZZ drafted the manuscript. DS and WZ interpreted the data and made a critical revision to the manuscript. MQ, DL, ZJ, MY contributed to data collection and performed the literature search. WZ provided consultation, participated in the coordination of the manuscript. All authors read and approved the final manuscript.

\section{Acknowledgements}

None.

\section{Authors' information}

${ }^{1}$ Department of Cardiology, China-Japan Union Hospital of Jilin University, Jilin Provincial Molecular Biology Research Center for Precision Medicine of Major Cardiovascular Disease, Changchun, China.

${ }^{2}$ Department of gastroenterology, endoscopy center, China-Japan Union Hospital of Jilin University, Changchun, Jilin, China.

\section{References}


1. Lattuca B, Bouziri N, Kerneis M, Portal JJ, Zhou J, Hauguel-Moreau M, Mameri A, et al. ACTION Study Group. Antithrombotic Therapy for Patients With Left Ventricular Mural Thrombus. J Am Coll Cardiol. 2020 Apr 14;75(14):1676-1685.

2. Delewi R, Nijveldt R, Hirsch A, Marcu CB, Robbers L, Hassell ME, et al. Left ventricular thrombus formation after acute myocardial infarction as assessed by cardiovascular magnetic resonance imaging. Eur J Radiol. 2012 Dec;81(12):3900-4.

3. Delewi R, Zijlstra F, Piek JJ. Left ventricular thrombus formation after acute myocardial infarction. Heart. 2012 Dec;98(23):1743-9.

4. Solheim S, Seljeflot I, Lunde K, Bjørnerheim R, Aakhus S, Forfang K, Arnesen H. Frequency of left ventricular thrombus in patients with anterior wall acute myocardial infarction treated with percutaneous coronary intervention and dual antiplatelet therapy. Am J Cardiol. 2010 Nov 1;106(9):1197-200.

5. Kalra A, Jang IK. Prevalence of early left ventricular thrombus after primary coronary intervention for acute myocardial infarction. J Thromb Thrombolysis. 2000 Oct;10(2):133-6.

6. Zielinska M, Kaczmarek K, Tylkowski M. Predictors of left ventricular thrombus formation in acute myocardial infarction treated with successful primary angioplasty with stenting. Am J Med Sci. 2008 Mar;335(3):171-6.

7. Bulluck H, Chan MHH, Paradies V, Yellon RL, Ho HH, Chan MY, et al. Incidence and predictors of left ventricular thrombus by cardiovascular magnetic resonance in acute ST-segment elevation myocardial infarction treated by primary percutaneous coronary intervention: a meta-analysis. $J$ Cardiovasc Magn Reson. 2018 Nov 8;20(1):72.

8. O'Gara PT, Kushner FG, Ascheim DD, Casey DE Jr, Chung MK, de Lemos JA, et al. American College of Cardiology Foundation/American Heart Association Task Force on Practice Guidelines. 2013 ACCF/AHA guideline for the management of ST-elevation myocardial infarction: a report of the American College of Cardiology Foundation/American Heart Association Task Force on Practice Guidelines. Circulation. 2013 Jan 29;127(4):e362-425.

9. Kernan WN, Ovbiagele B, Black HR, et al; American Heart Association Stroke Council, Council on Cardiovascular and Stroke Nursing, Council on Clinical Cardiology, and Council on Peripheral Vascular Disease. Guidelines for the prevention of stroke in patients with stroke and transient ischemic attack: a guideline for healthcare professionals from the American Heart Association/American Stroke Association. Stroke. 2014;45(7):2160-2236.

10. Ibanez B, James S, Agewall S, et al; ESC Scientific Document Group. 2017 ESC Guidelines for the management of acute myocardial infarction in patients presenting with ST-segment elevation: the task force for the management of acute myocardial infarction in patients presenting with STsegment elevation of the European Society of Cardiology (ESC). Eur Heart J. 2018;39(2):119-177.

11. Carpenter K, Adams D. Apical mural thrombus: technical pitfalls. Heart. 1998 Nov;80 Suppl 1(Suppl 1):S6-8. 
12. Schulman S, Kearon C; Subcommittee on Control of Anticoagulation of the Scientific and Standardization Committee of the International Society on Thrombosis and Haemostasis. Definition of major bleeding in clinical investigations of antihemostatic medicinal products in non-surgical patients. J Thromb Haemost. 2005 Apr;3(4):692-4.

13. Kaatz S, Ahmad D, Spyropoulos AC, Schulman S; Subcommittee on Control of Anticoagulation. Definition of clinically relevant non-major bleeding in studies of anticoagulants in atrial fibrillation and venous thromboembolic disease in non-surgical patients: communication from the SSC of the ISTH. J Thromb Haemost. 2015 Nov;13(11):2119-26.

14. Peduzzi P, Concato J, Feinstein AR, Holford TR. Importance of events per independent variable in proportional hazards regression analysis. II. Accuracy and precision of regression estimates. J Clin Epidemiol. 1995 Dec;48(12):1503-10.

15. Guddeti RR, Anwar M, Walters RW, Apala D, et al. Treatment of Left Ventricular Thrombus With Direct Oral Anticoagulants: A Retrospective Observational Study. Am J Med. 2020 Dec;133(12):1488-1491.

16. Robinson AA, Trankle CR, Eubanks G, Schumann C, Thompson P, Wallace RL, Gottiparthi S, Ruth B, Kramer CM, Salerno M, Bilchick KC, Deen C, Kontos MC, Dent J. Off-label Use of Direct Oral Anticoagulants Compared With Warfarin for Left Ventricular Thrombi. JAMA Cardiol. 2020 Jun 1;5(6):685-692. doi: 10.1001/jamacardio.2020.0652. PMID: 32320043; PMCID: PMC7177639.

17. Jones DA, Wright P, Alizadeh MA, Fhadil S, et al. The Use of Novel Oral Anti-Coagulant's (NOAC) compared to Vitamin K Antagonists (Warfarin) in patients with Left Ventricular thrombus after Acute Myocardial Infarction (AMI). Eur Heart J Cardiovasc Pharmacother. 2020 Jul 30:pvaa096.

18. Iqbal H, Straw S, Craven TP, Stirling K, Wheatcroft SB, Witte KK. Direct oral anticoagulants compared to vitamin $\mathrm{K}$ antagonist for the management of left ventricular thrombus. ESC Heart Fail. 2020 Oct;7(5):2032-2041.

19. Patel MR, Mahaffey KW, Garg J, Pan G, Singer DE, et al. ROCKET AF Investigators. Rivaroxaban versus warfarin in nonvalvular atrial fibrillation. N Engl J Med. 2011 Sep 8;365(10):883-91.

20. Gibson CM, Mehran R, Bode C, Halperin J, et al. Prevention of Bleeding in Patients with Atrial Fibrillation Undergoing PCI. N Engl J Med. 2016 Dec 22;375(25):2423-2434.

21. Lip GY, Hammerstingl C, Marin F, Cappato R, Meng IL, et al. X-TRA study and CLOT-AF registry investigators. Left atrial thrombus resolution in atrial fibrillation or flutter: Results of a prospective study with rivaroxaban (X-TRA) and a retrospective observational registry providing baseline data (CLOT-AF). Am Heart J. 2016 Aug; 178:126-34.

22. Seecheran R, Seecheran V, Persad S, Seecheran NA. Rivaroxaban as an Antithrombotic Agent in a Patient With ST-Segment Elevation Myocardial Infarction and Left Ventricular Thrombus: A Case Report. J Investig Med High Impact Case Rep. 2017 Mar 23;5(1):2324709617697991.

23. Mano Y, Koide K, Sukegawa $H$, Kodaira M, Ohki T. Successful resolution of a left ventricular thrombus with apixaban treatment following acute myocardial infarction. Heart Vessels. 2016 Jan;31(1):11823. 
24. Kao PH, Chou PY, Hsu PC, Huang TC. Resolution of left ventricular thrombus by edoxaban after failed treatment with warfarin overdose: A case report. Medicine (Baltimore). 2019 Jan;98(2):e14065.

25. Kajy M, Shokr M, Ramappa P. Use of Direct Oral Anticoagulants in the Treatment of Left Ventricular Thrombus: Systematic Review of Current Literature. Am J Ther. 2020 Nov/Dec;27(6):e584-e590.

26. Meltzer RS, Visser CA, Fuster V. Intracardiac thrombi and systemic embolization. Ann Intern Med. 1986 May;104(5):689-98.

27. Mega JL, Braunwald E, Murphy SA, Plotnikov AN, et al. Rivaroxaban in patients stabilized after a STsegment elevation myocardial infarction: results from the ATLAS ACS-2-TIMI-51 trial (Anti-Xa Therapy to Lower Cardiovascular Events in Addition to Standard Therapy in Subjects with Acute Coronary Syndrome-Thrombolysis In Myocardial Infarction-51). J Am Coll Cardiol. 2013 May 7;61(18):1853-9.

28. Eikelboom JW, Mehta SR, Anand SS, Xie C, Fox KA, Yusuf S. Adverse impact of bleeding on prognosis in patients with acute coronary syndromes. Circulation 2006;114:774-782.

29. Toyoda K, Yasaka M, Iwade K, Nagata K, et al. Bleeding with Antithrombotic Therapy (BAT) Study Group. Dual antithrombotic therapy increases severe bleeding events in patients with stroke and cardiovascular disease: a prospective, multicenter, observational study. Stroke. 2008 Jun;39(6):17405 .

30. Lamberts $\mathrm{M}$, Olesen JB, Ruwald MH, et al. Bleeding after initiation of multiple antithrombotic drugs, including triple therapy, in atrial fibrillation patients following myocardial infarction and coronary intervention: a nationwide cohort study. Circulation. 2012 Sep 4;126(10):1185-93.

31. Andrade JG, Deyell MW, Khoo C, Lee M, et al. Risk of bleeding on triple antithrombotic therapy after percutaneous coronary intervention/stenting: a systematic review and meta-analysis. Can $\mathrm{J}$ Cardiol. 2013 Feb;29(2):204-12.

32. Dewilde WJ, Oirbans T, Verheugt FW, Kelder JC, et al. WOEST study investigators. Use of clopidogrel with or without aspirin in patients taking oral anticoagulant therapy and undergoing percutaneous coronary intervention: an open-label, randomised, controlled trial. Lancet. $2013 \mathrm{Mar}$ 30;381(9872):1107-15.

\section{Figures}




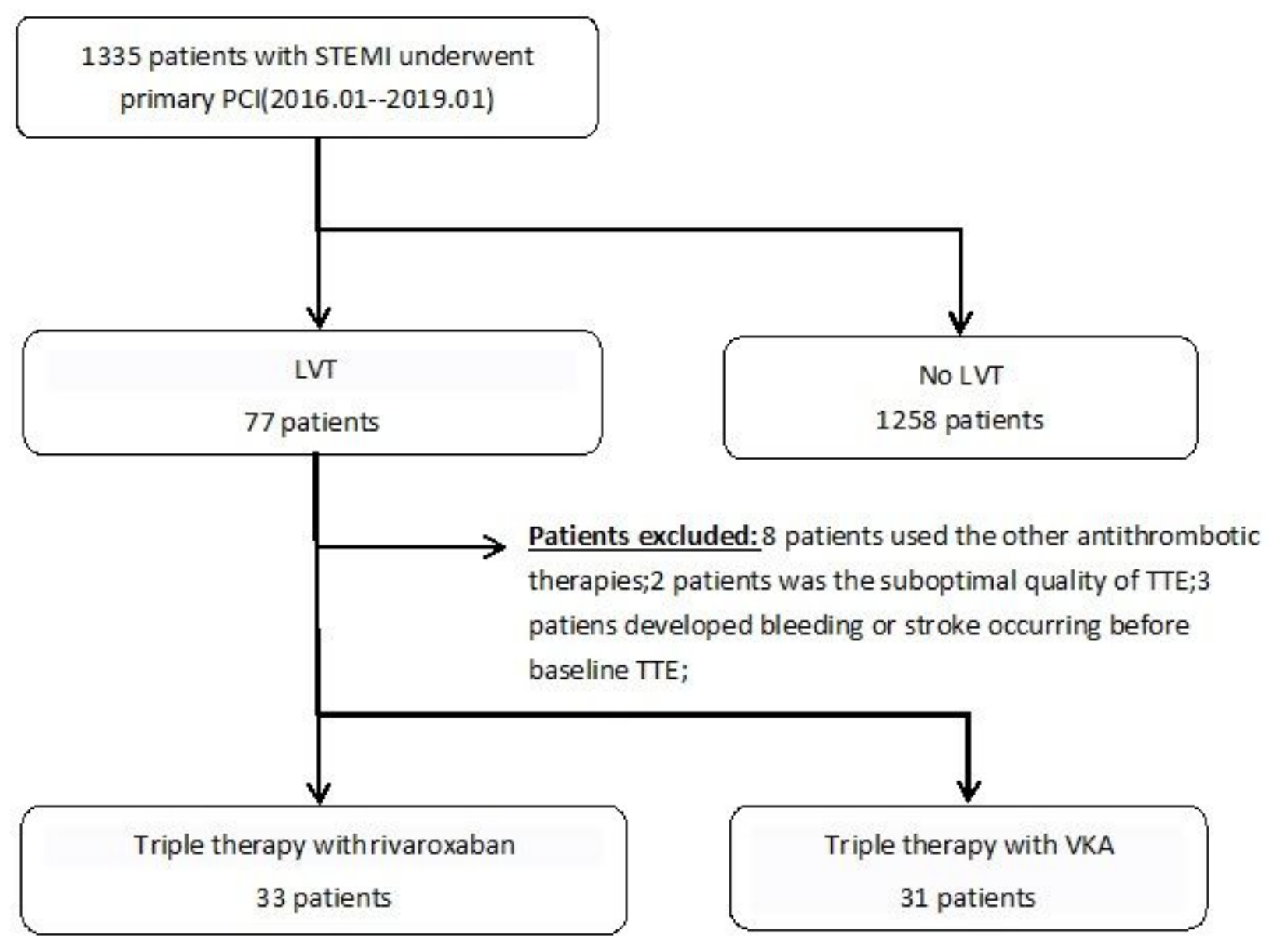

Figure 1

Study Chart Flow. STEMI:ST-Elevation Myocardial Infarction; PCI:percutaneous coronary intervention; LVT:Left ventricular thrombus;VKA:Vitamin K Antagonists 


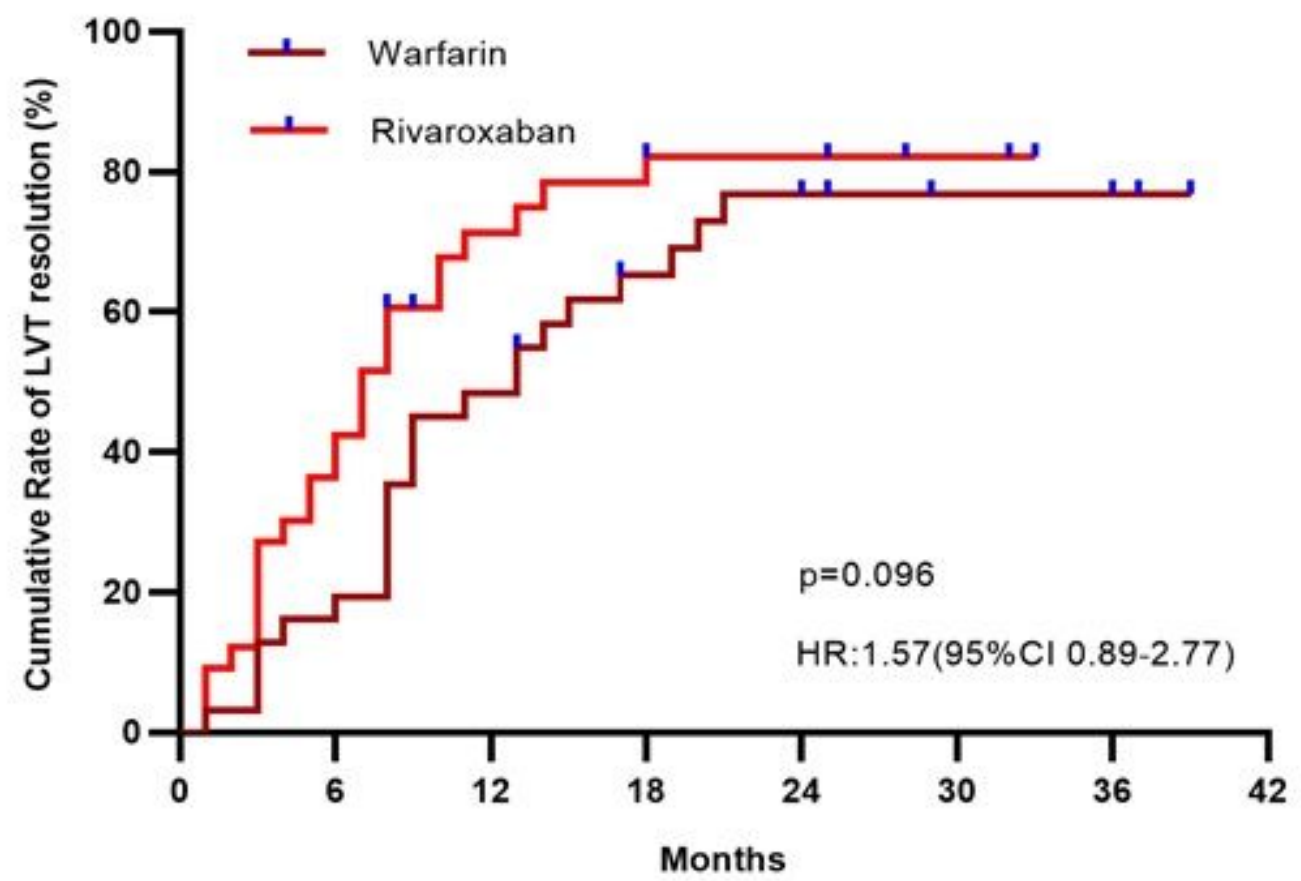

Figure 2

Time-to-Event Curves for LVT Resolution In Patients With Triple Therapy During The Entire Follow-up Period. LVT:Left ventricular thrombus;HR:hazard ratio; Cl:confidence interval;

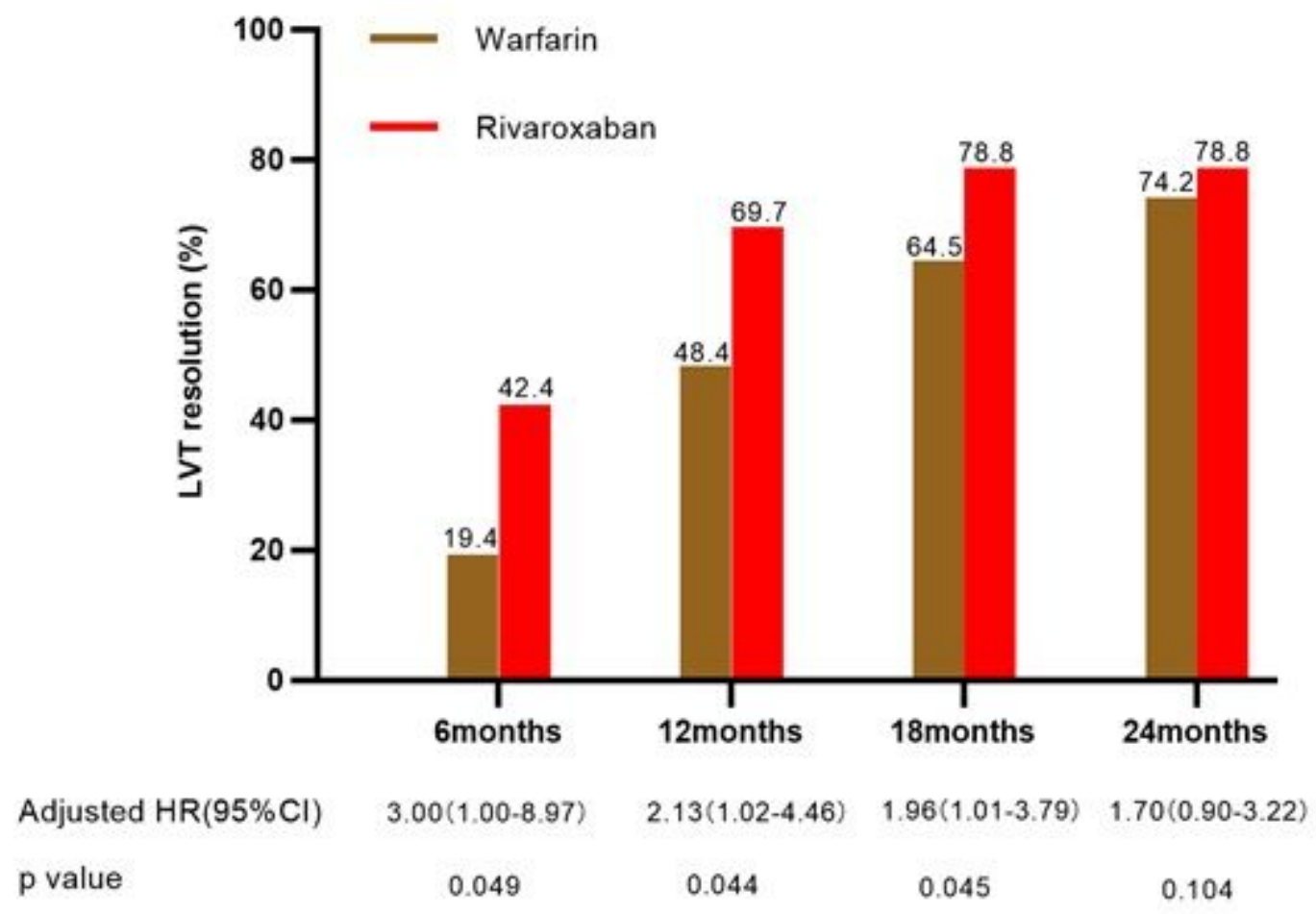

Figure 3 
LVT Resolution In Patients With Triple Therapy At Different Time Points During The Follow-up Period. LVT:left ventricular thrombus;HR:hazard ratio; Cl:confidence interval;

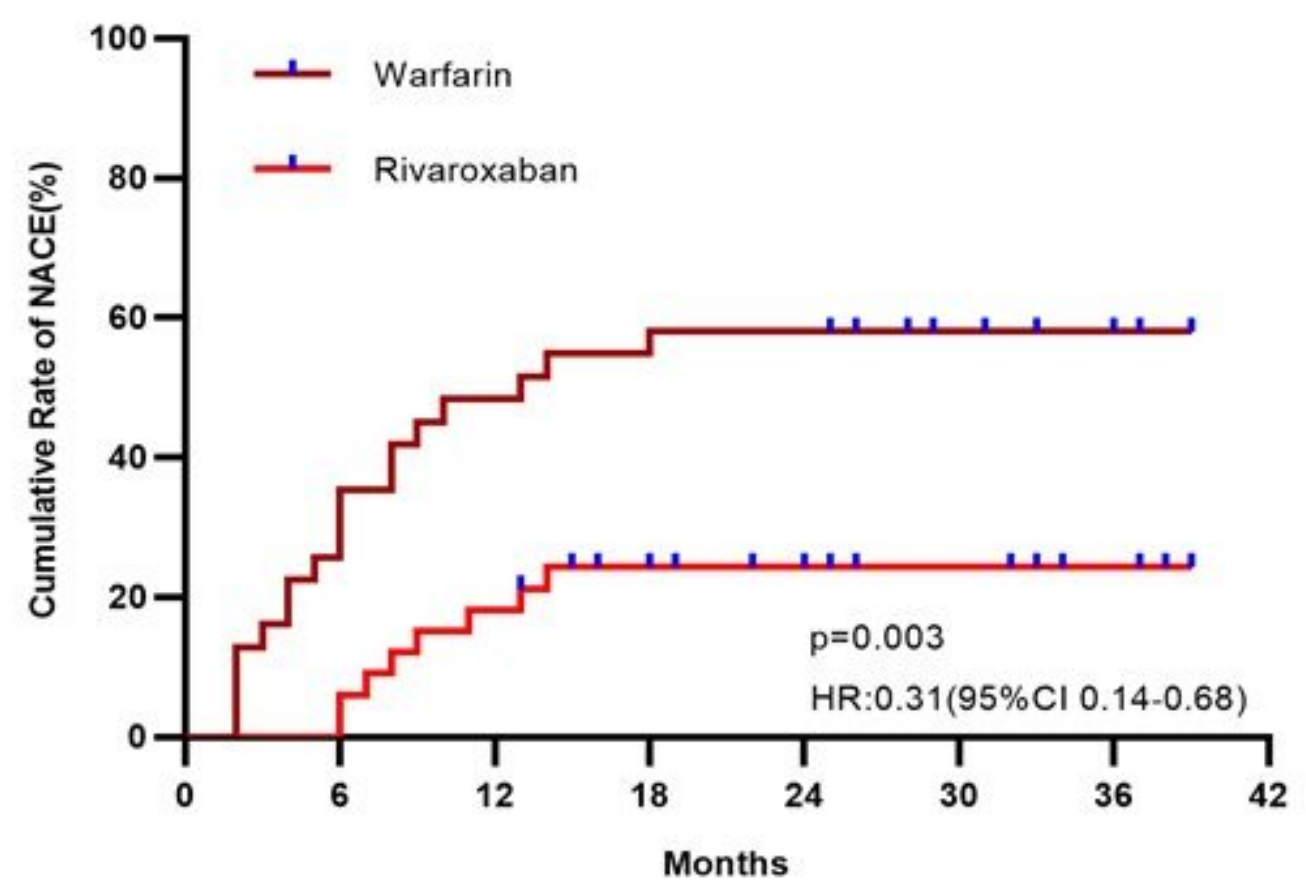

Figure 4

A composite of net adverse clinical events(NACE) consisting of all-cause mortality, SE ,rehospitalization for cardiovascular events, bleeding. HR:hazard ratio; Cl:confidence interval; 